\title{
Diffraction Effects in Terahertz Band - Measurements and Analysis
}

\author{
Joonas Kokkoniemi, Perttu Rintanen, Janne Lehtomäki, and Markku Juntti \\ Centre for Wireless Communications (CWC), \\ University of Oulu, P.O. Box 4500, 90014 Oulu, Finland \\ Email: joonas.kokkoniemi@ee.oulu.fi
}

\begin{abstract}
Measurement results and related analysis on the diffraction properties in the $\mathrm{THz}$ frequency band are reported. The results are given on the bands from approximately $100 \mathbf{G H z}$ to 2 THz. Measurements were made for knife-edge diffraction, and diffraction through single and double slits. The diffraction effects are of interest in modeling the non-line-of-sight (NLOS) paths in the THz band. Therefore, the measurement results have significant practical value for designing the real communication systems. The theoretical models from the literature show a very good match to the measurement results. The key finding is that wireless communication links in the THz band can be established even in the shadows of the objects through diffraction with reasonable path loss. This also indicates another path in the THz multipath environment, as the previous studies have shown the $\mathrm{THz}$ radiation is capable on penetration, reflections, and scattering.
\end{abstract}

\section{INTRODUCTION}

As more and more data traffic is generated every year, the demand for higher data rate links is ever increasing. The upcoming $5 \mathrm{G}$ systems are expected to utilize the millimeter wave frequencies (30-300 GHz) to seek more capacity over the exhausted ultrahigh frequency band $(300 \mathrm{MHz}-3 \mathrm{GHz})$ [1]. Moving to higher frequencies offers more bandwidth, but the path loss also unavoidably increases, too. This is perfectly in line with an intention to utilize smaller cells in, e.g., 5G to drastically increase the spatial throughput of the network through densification. The small cells can utilize the network resources better as there are less users in one cell in comparison to the modern systems.

Taking the data rates and small cell thinking a bit further, we arrive at the $\mathrm{THz}$ band $(0.1-10 \mathrm{THz})$. This band offers superior bandwidths to any lower frequency bands and it has been seen as a very potential band for short distance links and applications, such as indoor communications and nanocommunications [2]-[4]. With extremely high bandwidths, the theoretical data rates achieve and exceed one terabit-persecond [4]. The THz band's rapidly increasing popularity is partially caused by the appearance of solutions and materials to actually realize compact $\mathrm{THz}$ antennas and transceivers [5], [6]. The $\mathrm{THz}$ band has a problem with very high path loss, increased by, e.g., the molecular absorption caused (mostly) by the water molecules in the air [4], [7]. This causes the communication distances to remain modest, e.g., a few meters with directional antennas. Despite the high path loss, the $\mathrm{THz}$ band systems will be realized in the future. A big piece to make this happen is to theoretically and empirically study the channel in order to estimate the true potential of the $\mathrm{THz}$ band.

For this reason, we study one possible non-line-of-sight (NLOS) propagation phenomenon in this paper, the diffraction. The $\mathrm{THz}$ band is usually considered to require line-of-sight (LOS) due to large losses in the channel. However, we (and other groups) have shown in the past, that there exists multiple NLOS propagation phenomena, such as reflections, diffuse scattering, and penetration [8]-[10]. Adding the diffraction among these phenomena, we potentially have quite a rich multipath environment in the THz band. However, the NLOS paths have significantly less power in comparison to the LOS path, as it will also be shown for diffraction.

Two diffraction measurement cases are studied, the knifeedge diffraction and slit diffraction. The knife-edge diffraction is a very practical problem because of the objects in the propagation path may shadow the receiver from the transmitter. The slit diffraction is more rare in the communication channels, but perfectly possible. The slit may be caused, e.g., by a partially open door, etc. Theoretical models for the both cases are given and compared against the measurement data. The results show that the theories agree very well with the measurements, although, the knife-edge theory had to be modified with an additional term for the knife-edge with finite thickness.

The knife-edge diffraction has been studied previously in [11], [12] for the lower THz band (0.30-0.31 THz). Those studies also showed good agreement of the measurement data to the theories. We measure with a much larger band by utilizing the time domain spectroscopy for the channel sounding (0.1-2.0 THz) with TeraView TeraPulse 4000 measurement device. The measurement setup will be detailed in Section III.

The rest of this paper is organized as follows. In Section II, the basic theories behind the diffraction phenomena are given. Section III presents the utilized measurement setups. Numerical results are given in Section IV and Section V concludes the paper.

\section{DiFFRACTION THEORY}

All the theories on the diffraction phenomena are very well established. In the theories, we assume ideal materials and measurement setups, except for the knife-edge theory, which required a minor adjustment because of the finite knife-edge width. Surprisingly, and as it can be seen the numerical results 
in Section IV, even these simple forms of the theories provide a very good fit to the experimental results.

\section{A. Knife-Edge Diffraction}

The knife-edge diffraction is caused by an obstacle, and especially the edge of the obstacle operating as a secondary source for the radiation. This allows the radiation to reach the shadow region of the obstacle. The knife-edge path gain is described by [11]-[15]

$$
L(\theta)=\frac{(1-C(\nu)-S(\nu))^{2}+(C(\nu)-S(\nu))^{2}}{4},
$$

where $\theta$ is the diffraction angle given in Fig. $1, C(\nu)$ and $S(\nu)$ are the Fresnel integrals given by

$$
\begin{aligned}
& C(\nu)=\int_{0}^{\nu} \cos \left(\frac{\pi s^{2}}{2}\right) d s, \\
& S(\nu)=\int_{0}^{\nu} \sin \left(\frac{\pi s^{2}}{2}\right) d s,
\end{aligned}
$$

and $\nu$ is the Fresnel parameter given by

$$
\nu=h \sqrt{\frac{2}{\lambda}\left(\frac{1}{d_{1}}+\frac{1}{d_{2}}\right)},
$$

where $h$ is the height of the knife-edge measured from the LOS path as shown in Fig. 1, and $\lambda$ is the wavelength. Based on our measurement setup detailed in the next section, we can calculate the length of the LOS path $x$ (see Fig. 1) with the law of cosines as

$$
x=\sqrt{d_{2}^{2}+d_{1}^{2}-2 d_{1} d_{2} \cos (\pi-\theta)} .
$$

From the LOS path length we get the angle $\alpha$ as

$$
\alpha=\cos ^{-1}\left(\frac{d_{1}^{2}+x^{2}-d_{2}^{2}}{2 x d_{1}}\right) .
$$

Finally, we get the effective height $h$ of the knife-edge with

$$
h=d_{1} \tan (\alpha) .
$$

In the actual measurement setup we utilize a knife-edge of finite thickness. This causes the above theoretical prediction to differ from the measurement results. The finite thickness leads to more attenuation in the shadow region of the diffraction. Therefore, we utilize an additional attenuation introduced by a round obstacle. This was noticed to rectify the theoretical model towards the measured values, although, the knifeedge in the measurement was rectangular. The edge in the measurements can be thought as two consecutive infinitely sharp knife-edges beside each other, separated by a distance $2 R$, where $R$ is a diameter of an imagined circle between the edges. Thus, it is a half of the knife-edge thickness. With this assumption the additional loss to the ideal knife-edge in $\mathrm{dB}$-scale is given by [14]

$$
T_{\mathrm{dB}}(m, n)=7.2 m^{1 / 2}-(2-12.5 n) m+3.6 m^{3 / 2}-0.8^{2},
$$

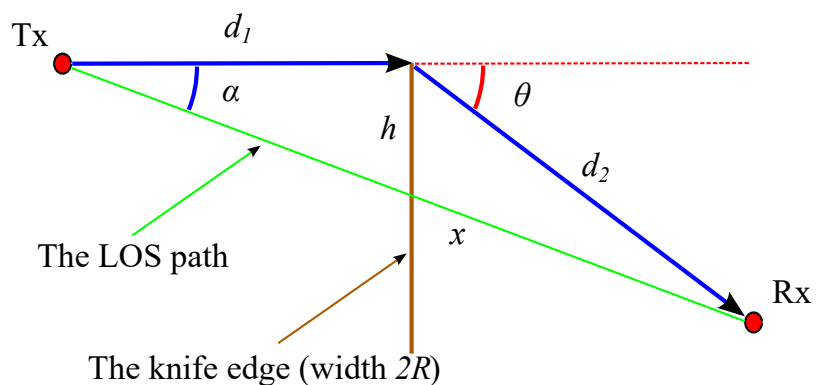

Fig. 1. The system geometry for the knife-edge measurements. The same setup applies for the angles also in the slit diffraction measurements.

for $m n \leq 4$, and

$$
\begin{aligned}
T_{\mathrm{dB}}(m, n)= & -6-20 \log _{10}(m n)+7.2 m^{1 / 2} \\
& -(2-17 n) m+3.6 m^{3 / 2}-0.8^{2},
\end{aligned}
$$

for $m n>4$, where

$$
m=R\left(\frac{1}{d_{1}}+\frac{1}{d_{2}}\right) /\left(\frac{\pi R}{\lambda}\right)^{1 / 3},
$$

and

$$
n=\frac{h}{R}\left(\frac{\pi R}{\lambda}\right)^{2 / 3}
$$

Then the total path gain (in linear scale) is given by

$$
L_{\text {tot }}(\theta)=L(\theta) 10^{-T_{\mathrm{dB}}(m, n) / 10} \text {. }
$$

\section{B. Slit Diffraction}

The slit diffraction is very well known and familiar from optics. The diffraction pattern is caused by the slit is acting as a "virtual" source for the radiation (similarly as in the case of knife-edge, where the edge is acting as a source). In the case of multiple slits, multiple sources causes more defined diffraction pattern as it will be shown in the numerical results in Section IV. We also see in the numerical results that the standard slit diffraction theories match very well with the measured values.

The slit diffraction theory for a single slit is given by $[16$, Chap. 36.3]

$$
\frac{I}{I_{0}}=\left(\frac{\sin (\beta / 2)}{\beta / 2}\right)^{2},
$$

where $I$ is the received radiation intensity, $I_{0}$ is the incident radiation intensity, and

$$
\beta=\frac{2 \pi a}{\lambda} \sin (\theta)
$$

where $a$ is the slit width, and $\theta$ is the diffraction angle (as in above, and in Fig. 1).

The slit diffraction attenuation for a double slit is given by [16, Chap. 36.4]

$$
\frac{I}{I_{0}}=\cos \left(\frac{\phi}{2}\right)^{2}\left(\frac{\sin (\beta / 2)}{\beta / 2}\right)^{2},
$$

where

$$
\phi=\frac{2 \pi d}{\lambda} \sin (\theta)
$$

where $d$ is the slit separation distance. 


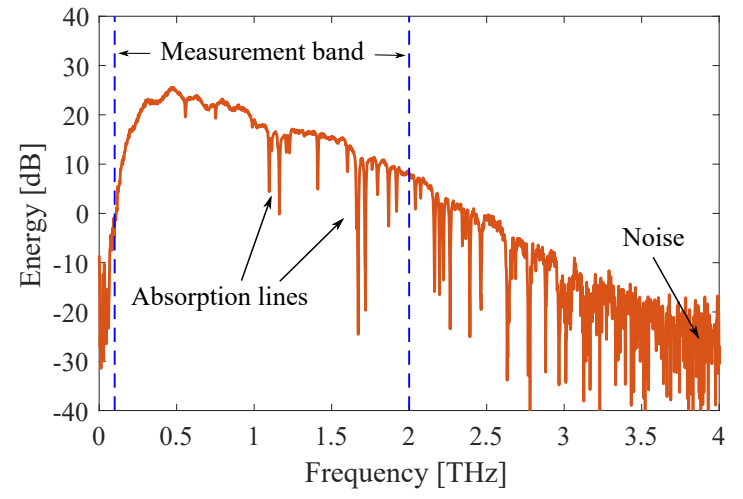

Fig. 2. The pulse shape of the TeraView TeraPulse showing the exponentially decaying amplitude/energy characteristics at the lower and higher extreme frequencies, as well as the measurement band for which the results are given.

TABLE I

MEASUREMENT SETUP AS NUMBERS.

\begin{tabular}{|c|c|}
\hline \hline Quality & Quantity \\
\hline \hline Averaging & 100 measurements per angle \\
Detection window & $200 \mathrm{ps}$ \\
Frequency resolution & $5 \mathrm{GHz}$ \\
Time resolution & $100 \mathrm{fs}$ \\
Distance $d_{1}$ & $10 \mathrm{~cm}$ \\
Distance $d_{2}$ & $10 \mathrm{~cm}$ \\
Radius $R$ & $0.1 \mathrm{~cm}$ \\
Slit width $a$ & $2 \mathrm{~mm}$ \\
Slit separation $d$ & $2 \mathrm{~mm}$ \\
\hline
\end{tabular}

\section{Measurement Setup}

The measurements were conducted with TeraView TeraPulse 4000 (MiniPulse) measurements device. This device is capable of measuring frequencies from approximately 0.1 to 4 THz. The lower and the higher frequencies are limited by the pulse shape, having an exponentially increasing end decreasing shape with a peak amplitude at around $500 \mathrm{GHz}$. The pulse shape in frequency domain is shown in Fig. 2. The figure also shows the effective measurement band limited by the weak signal components not being visible after the diffraction. The molecular absorption lines can be seen as deeply faded frequencies in the spectrum. After about $4 \mathrm{THz}$, the signal is completely under the noise level.

The measurements are based on terahertz time domain spectroscopy (THz-TDS). This method exploits the large bandwidth of very short pulses. As the name suggests, the signal detection occurs in the time domain. Each detected pulse is a composition of thousands of transmitted pulses (depending on the measurement setup). To detect the entire pulse the device utilizes a delay line, allowing a detection of the pulse one time instant at the time. The device sends a pulse and the receiver end measures the response at the certain time instant. After the detection, it moves to measure the next time instant. Following this procedure, the entire time grid can be measured and detected after sending $T_{d} / d t$ pulses, where $T_{d}$ is the detection window length, and $d t$ is the time resolution of the time grid. With the measurement setup in this paper, the number of samples per pulse was 2000. The other metrics of

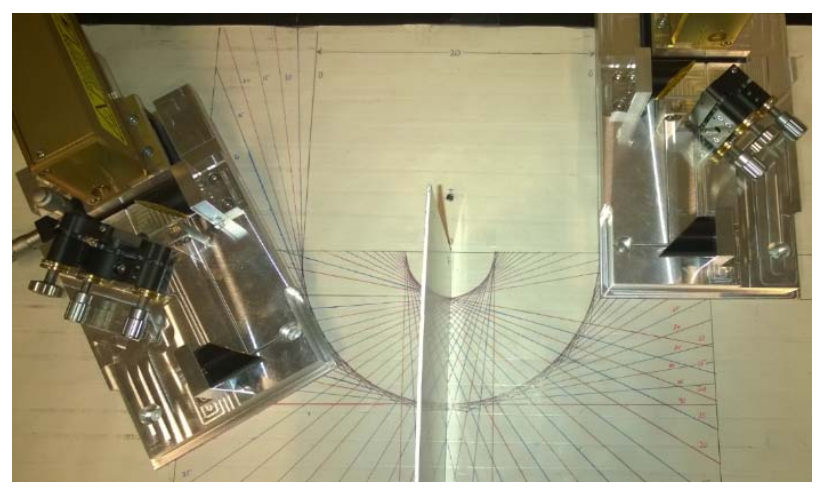

Fig. 3. The measurement setup for knife-edge measurements. The receiver (left-hand plate) is at 30 degree angle to the knife-edge.

the measurement device used in these measurements are given in Table I.

The measurement device uses optics to focus the beam at the sample location, which is between the plates holding the transmitter and receiver heads, as well as the optics. The optics are visible, e.g., in Fig. 3. Between the plates, the beam is nearly perfectly straight with approximately two centimeter diameter. The reason for focusing is to minimize the energy loss because of the signal spreading and as a consequence, to maximize the received energy and the energy at the sample.

\section{A. Knife-Edge Measurement Setup}

The knife-edge effect was measured with the measurement setup shown in Fig. 3. A two millimeter thick aluminium sheet was placed at the center of the beam, at ten centimeter distance from the emitter (right-hand side measurement head). Thus, $R=0.1 \mathrm{~cm}$ was used in Eqs. (10) and (11). The receiver was then moved with five degree intervals from zero degree to 70 degrees keeping the distance fixed to the center point. From the theory point of view, angle $\theta$ was variable, whereas the distances $d_{1}$ and $d_{2}$ were fixed at ten centimeters (see Fig. 1).

A two millimeter thick aluminium sheet was chosen as the knife-edge as it is perfectly opaque at the $\mathrm{THz}$ frequencies while being relatively thin. However, in the THz wavelengths, the two millimeter thickness caused the simplest knife-edge theory to fail, requiring the assumption on the round knifeedge presented in the previous section. As it was mentioned, this assumption turned out to accurately predict the knife-edge effect in the THz band. Similar behavior can be expected with most of the other materials as well. In some cases, such as with very thick samples, the double knife-edge theory [14] may be worth considering in order to predict the diffracted intensities.

\section{B. Slit Diffraction Measurement Setup}

The measurement setup for the slit diffraction is shown in Fig. 4. The slit screens were composed of copper plated fiber class (a blank circuit board). The copper plating made sure the material did not allow $\mathrm{THz}$ radiation to pass through, which was also confirmed with measurements. The figure shows the two-slit screen. Illustrations on the slit screens for the single 


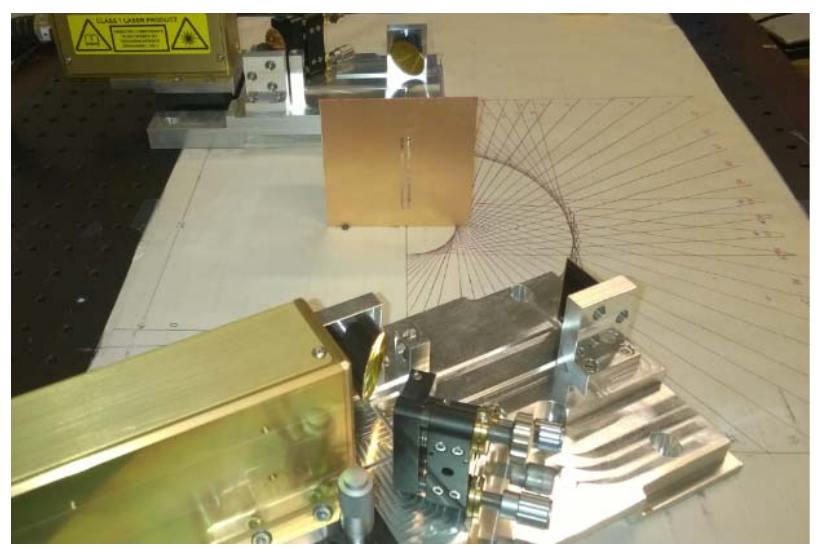

Fig. 4. The measurement setup for slit diffraction measurements. The two slit sample is in place for this figure and the receiver is at 30 degree angle to the sample. (a) Single slit

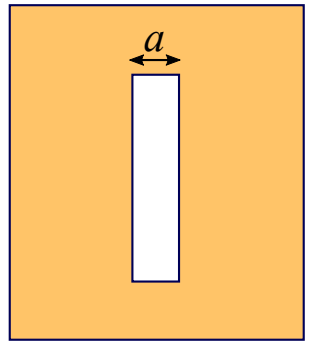

(b) Double slit

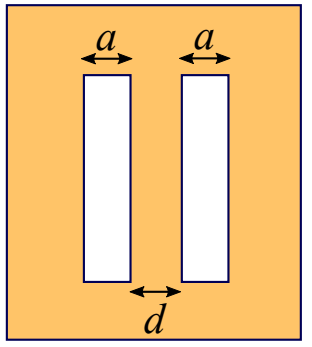

Fig. 5. Illustrations on the slit configurations utilized in the measurements showing the slit width $a$ and slit separation distance $d$.

and double slit measurement cases are also given in Fig. 5 . The slit width $a$ was two millimeters in both cases to ensure a good diffraction pattern at the $\mathrm{THz}$ band (tested with the theories prior to making the samples). In the case of two slits, the slit separation $d$ was two millimeters as well. The measured diffraction angle was varied similarly as in the knifeedge measurements, and similarly, the distances from the $\mathrm{THz}$ emitter and receiver to the sample were kept fixed at ten centimeters.

\section{NumericAl RESULtS}

The measurement results and comparisons to respective theories are shown in this section. The knife-edge is looked into first followed by the slit diffraction measurements.

\section{A. Knife-Edge Measurement Results}

Figure 7(a) shows the measured knife-edge path gains (dB) as a function of frequency and diffraction angle. The path gain was obtained by comparing the measurement results against a reference signal measured at full LOS (e.g., shown in Fig. 2). The theoretical path gain is given by (12), and shown in Fig. 7(b) for the parameters discussed in the theory section and reflecting the measurement setup given in the previous section. We can see the measurement results agree very well with the theory for the round knife-edge path gain given in (12). Both the theory and the measurements are suppressed to give the minimum path gain of $-70 \mathrm{~dB}$ in order

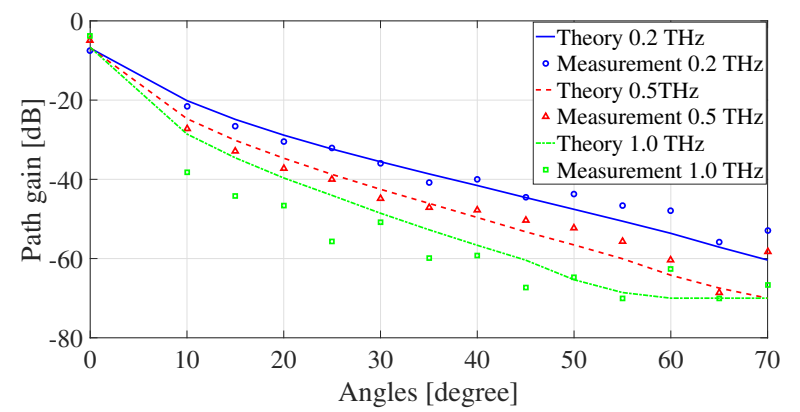

Fig. 6. Theoretical and measured knife-edge effect responses at the receiver as a function of diffraction angle at $0.2,0.5$, and $1.0 \mathrm{THz}$ frequencies.

to focus on the transmission in the shadowed regions. As it was discussed in the last section, the measurement results for the frequency bands below $100 \mathrm{GHz}$ are not utilizable due to pulse shape. Otherwise the theory and the empirical data converge. Another angle on the results is given in Fig. 6, where the path gains are given for three frequencies as a function of the diffraction angle. Also this figure shows great fit between the measurement data and the theoretical model.

The excellent fit is an expected result as the theories are fundamental results for any frequency band. At the same time, this is an interesting result from the viewpoint of multiple possible NLOS propagation methods for the $\mathrm{THz}$ band communications. We have previously shown the existence of the scattering/reflection NLOS response at receiver [8], [9] (similarly as, e.g. in [17]-[19]), as well as the NLOS contribution after penetration [10]. Adding to these a very interesting contribution provided by the diffraction, offering a possibility of reception in the shadow region, we start to have quite an interesting multi-path environment in the $\mathrm{THz}$ band. However (as expected), the diffraction response quickly goes near the noise level as the angle is increased. Therefore, the expected operational region for communication is at shallow angles behind the objects. As it can be seen in Fig. 6, 10 degree angle is still giving reasonable path gain, but the response decays exponentially as the diffraction angle is increased.

The knife-edge is of particular interest, as it is in effect, e.g., when a table is blocking a receiver from a transmitter sitting on the table. Next we will go to the slit diffraction, more rare phenomenon in the communication systems, but interesting from the scientific point of view. However, there are applications for the slit diffraction as well.

\section{B. Slit Diffraction Measurement Results}

The slit diffraction measurement data for the path loss (dB) in one-slit case is given in Fig. 7(c), and for the twoslit case in Fig. 7(e). The corresponding theoretical values are given in Figs. 7(d) and 7(f). The figures are limited to have a minimum path gain of $-50 \mathrm{~dB}$ to better show the diffraction patterns. As it can be seen, the measurement data is similar to the theoretical values. The measurement data is more noisy and the finite beam diameter distorts the data, because the two centimeter wide beam allows reception at multiple angles at the same time. The measurement results 


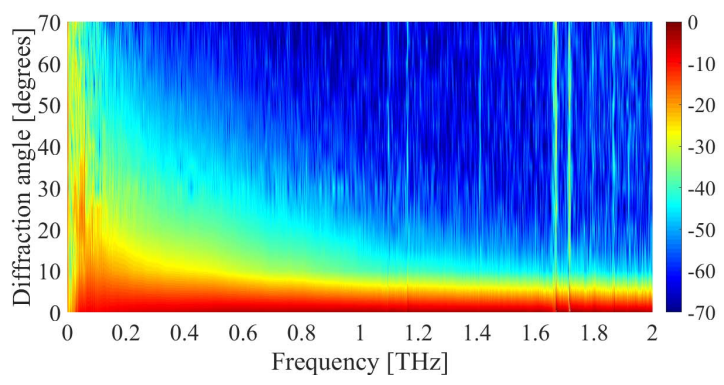

(a) Measured knife-edge effect.

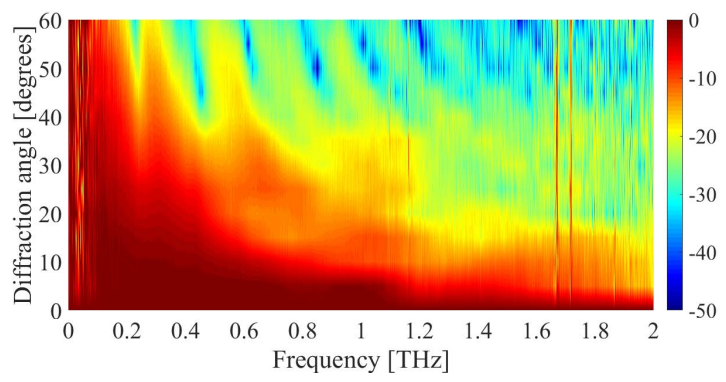

(c) Measured single slit effect.

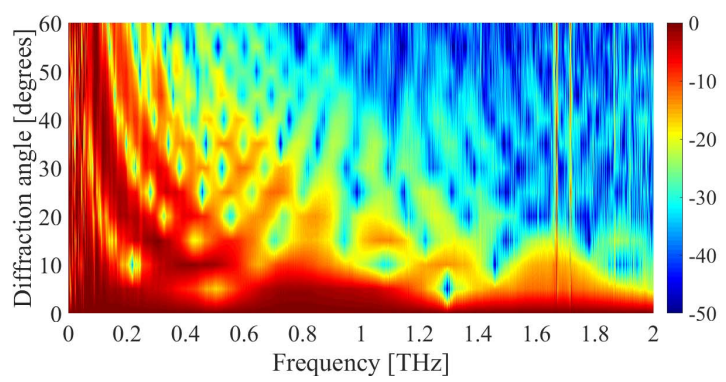

(e) Measured double slit effect.

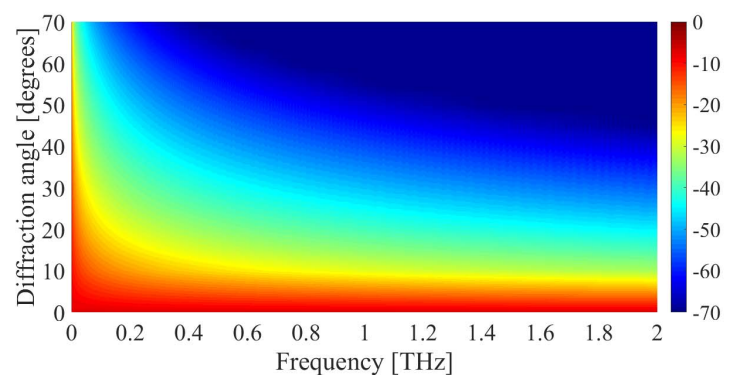

(b) Theoretical knife-edge effect.

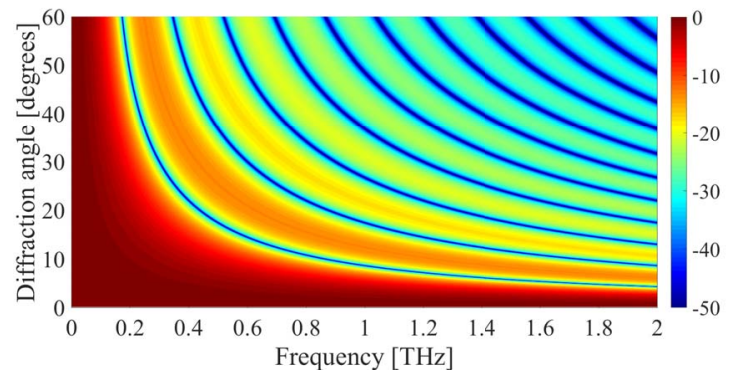

(d) Theoretical single slit effect.

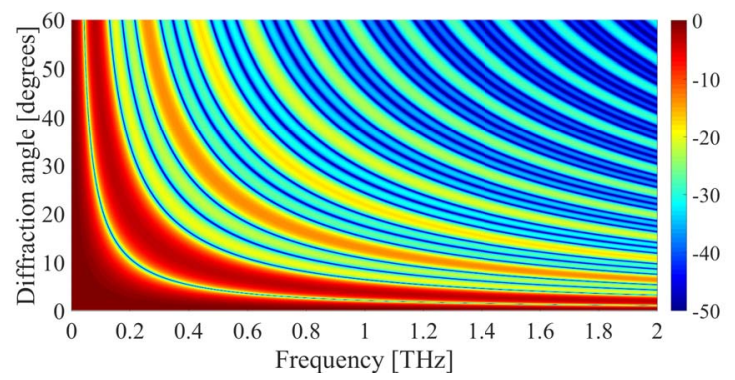

(f) Theoretical double slit effect.

Fig. 7. Measured (left-hand column) and theoretical (right-hand column) path gains in dB-scale at the receiver as a function of frequency and diffraction angle for the different diffraction effects.

are effectively obtained after two dimensional filter (due to the beam diameter), leading to blurry results. A part of the distortion comes from the non-ideal measurement setup. However, the corresponding diffraction features can be seen. Furthermore, attenuation at the maxima are almost spot on within tolerable error range. This can also be seen in Figs. 8 and 9, which show the frequency response of the single and double slit cases, respectively, for 40 degree diffraction angle. High angle was chosen to better illustrate the diffraction pattern, which is quite distorted in the lower angles.

The two-slit case suffers visually a little bit from the interpolation necessary to make the figure informative. It makes a false secondary grid opening from bottom left to upper right. This is in reality not in the data, but is an artifact by the interpolation (and can be seen in Figs. 8 and 9). Second issues with the two-slit case is the measurement data, which shows more like a single slit pattern. The maxima are in their correct places, but the maxima strengths are more uniform when comparing to the theoretical values. They show a similar pattern to the single slit case (Fig. 7(d)). This can also be seen in Fig. 9. This requires further investigations in the future.
However, the most probable reason lies in the previously mentioned finite beam diameter, hiding the fine structure of the diffraction pattern. The reason may also a similar effect seen in the case of the knife-edge diffraction: the finite depth of the sample causes some unexpected behavior. However, the two slit diffraction is not probable in the real communication systems and the knife-edge effect is the most important with respect to modeling the NLOS paths in the $\mathrm{THz}$ band.

The overall picture of the slit diffraction shows lower attenuation to the knife-edge effect, with somewhat reasonable losses throughout the studied band. This is especially true for the single slit case, which has rather narrow minima. The two slit case widens the minima and therefore causes larger loss in the shadow region. Still, both of the slit diffraction cases would allow communication to the shadow region, given the transmit power is high enough.

\section{CONCLUSION}

We showed results for the $\mathrm{THz}$ band diffraction measurements. The empirical knife-edge diffraction showed possibility to communicate behind a corner in NLOS conditions. 


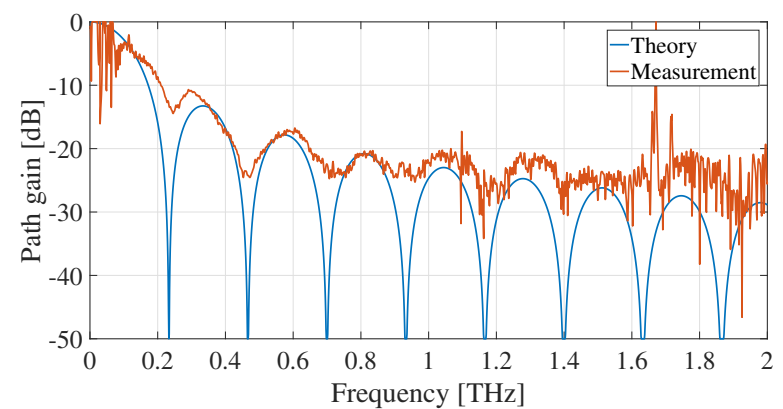

Fig. 8. Theoretical and measured single slit diffraction response at the receiver as a function of frequency at 40 degree diffraction angle.

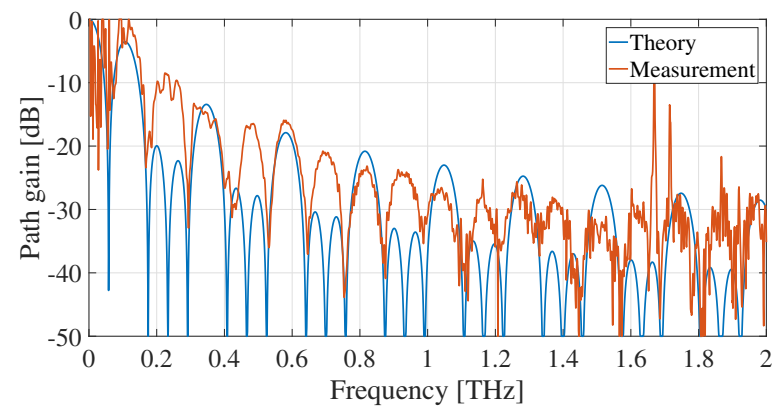

Fig. 9. Theoretical and measured double slit diffraction response at the receiver as a function of frequency at 40 degree diffraction angle.

Furthermore, we showed measurement results for the slit diffractions. This occurs rarely in real communication systems, but is perfectly feasible through narrow openings, e.g., a semi-closed door. Both the measurements and theory show weak response in the shadow region. However, a loss ranging between 5 and $50 \mathrm{~dB}$, depending on the diffraction angle and the phenomenon, theoretically allows communication in the shadow region of the objects through the diffracted rays.

The results encourage considering the diffraction as yet another multipath component in the $\mathrm{THz}$ communication channel. Earlier works have empirically shown existence of the penetration, reflection, and scattering contributions in the $\mathrm{THz}$ regime. Each of these effects causes time delayed response at the receiver due the path length differences as well as the dielectric differences in the propagation paths. Similarly, the diffraction introduces a delayed response at the receiver because of the increased path length. Therefore, the NLOS effects potentially cause additional interference to the coexisting links and possibly inter-symbol interference (ISI). This is because of the tight detection window length requirements set by the short pulses in the THz band, causing the NLOS paths to very likely spread the symbol energy over multiple symbols. Still, the contribution of the multipath components depends on several factors, such as, is the desired/interfered link in LOS or NLOS condition, as well on the distances and the antenna directivities.

The overall picture shows the $\mathrm{THz}$ band offers much richer multipath environment than many, including we, have anticipated. This also causes a need for further investigations in the future to show the true face of the $\mathrm{THz}$ band as a communication channel.

\section{ACKNOWLEDGEMENT}

This work was supported by Infotech Oulu Doctoral Program and Academy of Finland under SeCoFu project. The first author would also like to thank the following foundations for their support: Riitta ja Jorma J. Takasen säätiö, Walter Ahlströmin säätiö, Tauno Tönningin säätiö, Ulla Tuomisen säätiö, and Nokia Foundation.

\section{REFERENCES}

[1] T. S. Rappaport et al., "Millimeter wave mobile communications for 5G cellular: It will work!" IEEE Access, vol. 1, no. 1, pp. 335-349, May 2013.

[2] I. F. Akyildiz, J. M. Jornet, and C. Han, "Terahertz band: Next frontier for wireless communications," Phys. Commun., vol. 12, pp. 16-32, Sep. 2014.

[3] I. F. Akyildiz and J. M. Jornet, "Electromagentic wireless nanosensor networks," Nano Commun. Networks J. (Elsevier), vol. 1, no. 1, pp 3-19, Mar. 2010.

[4] J. M. Jornet and I. F. Akyildiz, "Channel modeling and capacity analysis for electromagentic nanonetworks in the terahertz band," IEEE Trans. Wireless Commun., vol. 10, no. 10, pp. 3211-3221, Oct. 2011.

[5] — "Graphene-based plasmonic nano-antenna for terahertz band communication in nanonetworks," IEEE J. Sel. Areas Commun., vol. 31, no. 12 , pp. $685-694$, Dec. 2013

[6] _ , "Graphene-based plasmonic nano-transceiver for terehertz band communicaiton," in Proc. European Conf. Ant. Prop., 2014, pp. 492496.

[7] S. Paine, "The am atmospheric model," Smithsonian Astrophysical Observatory, Tech. Rep. 152, 2012.

[8] J. Kokkoniemi, J. Lehtomäki, and M. Juntti, "Measurements on rough surface scattering in terahertz band," in Proc. European Conf. Antennas Propag., 2016, pp. 1-5.

[9] J. Kokkoniemi, J. Lehtomäki, V. Petrov, D. Moltchanov, and M. Juntti, "Frequency domain penetration loss in the terahertz band," in Proc. Global Symb. Milli. Waves, 2016, pp. 1-4.

[10] J. Kokkoniemi, J. Lehtomäki, and M. Juntti, "Measurements on penetration loss in terahertz band," in Proc. European Conf. Antennas Propag., 2016, pp. 1-5.

[11] S. Priebe, C. Jastrow, M. Jacob, T. Kleine-Ostmann, T. Schrader, and T. Kürner, "Channel and propagation measurements at $300 \mathrm{GHz}$," IEEE Trans. Antennas Propag., vol. 59, no. 5, pp. 1688-1698, May 2011.

[12] M. Jacob, S. Priebe, R. Dickhoff, T. Kleine-Ostmann, T. Schrader, and T. Kürner, "Diffraction in $\mathrm{mm}$ and sub-mm wave indoor propagation channels," IEEE Trans. Microw. Theory Techn., vol. 60, no. 3, pp. 833844, Mar. 2012.

[13] M. Hall, L. Barclay, and M. Hewitt, Eds., Propagation of Radiowaves. Inspec/Iee, 1996.

[14] Recommendation ITU-R P.526-13: Propagation by diffraction, International Telecommunication Union Radiocommunication Sector (ITU-R) Std.

[15] M. Rieche, A. Ihlow, T. Heyn, F. P'rez-Font'n, and G. Del Galdo, "Land mobile satellite propagation characteristics from knife-edge diffraction modeling and hemispheric images," in Proc. European Antennas Propag., 2015, pp. 1-4.

[16] H. D. Young and R. A. Freedman, Sears and Zemansky's University Physics: with Modern Physics (11th ed.), A. Black, Ed. Addison Wesley, 2003.

[17] C. Jansen, S. Priebe, C. Möller, M. Jacob, H. Dierke, M. Koch, and T. Kürner, "Diffuse scattering from rough surfaces in $\mathrm{THz}$ communication channels," IEEE Trans. THz Sci. Technol., vol. 1, no. 2, pp. 462-472, Nov. 2011.

[18] S. Priebe, M. Jacob, C. Jansen, and T. Kürner, "Non-specular scattering modeling for THz propagation simulations," in Proc. IEEE European Conf. Antennas Propag., 2011, pp. 1-5.

[19] R. Piesiewicz, C. Jansen, D. Mittleman, T. Kleine-Ostmann, M. Koch, and T. Kürner, "Scattering analysis for the modeling of $\mathrm{THz}$ communication systems," IEEE Trans. Antennas Propag., vol. 55, no. 11, pp. 3002-3009, Nov. 2007 